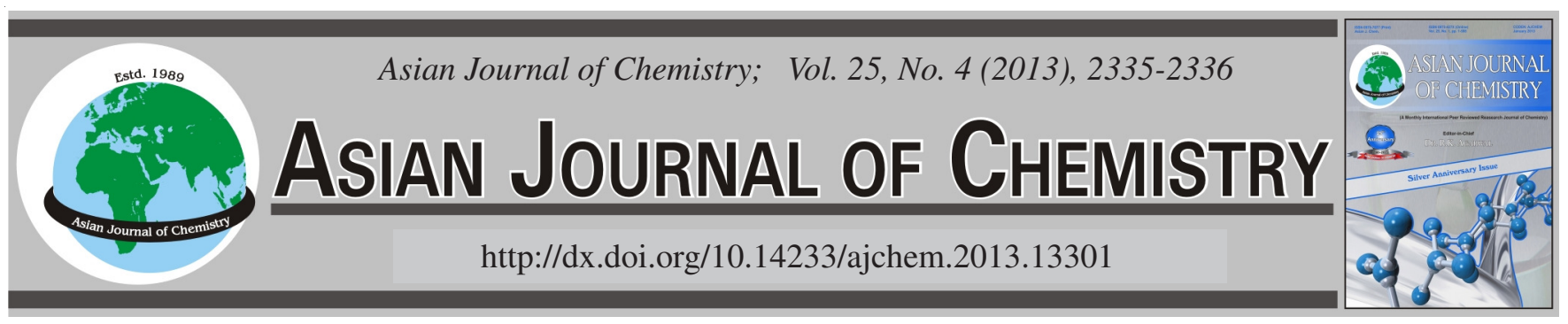

NOTE

\title{
One New Flavonoid Glycoside from Chrysanthemum morifolium
}

\section{Weiming Cheng ${ }^{1, *}$, Xiagian Cheng ${ }^{2}$, Yongchi Zeng ${ }^{3}$ and Wenting Zhang ${ }^{1, *}$}

${ }^{1}$ Department of Traditional Chinese Medicine, Zhejiang Institute for Food and Drug Control, No. 86 Lane 1, Jichang Road, Jianggan Dist. 310004, Hangzhou Zhejiang Province, P.R. China

${ }^{2}$ College of Pharmaceutical Science, Zhejiang Chinese Medicinal University, No 548 Bingwen Road, Binjiang Dist. 310053, Hangzhou Zhejiang Province, P.R.China

${ }^{3}$ Hangzhou Innovation TCM Standarization Research Institute Co. Ltd., No 677 Binkang Road, Binjiang Dist. 310053, Hangzhou Zhejiang Province, P.R. China

*Corresponding author: Tel: +86 158 58177067; E-mail: weiming_cheng@hotmail.com

(Received: 21 December 2011;

Accepted: 29 October 2012)

AJC-12348

\begin{abstract}
The investigation of water-soluble fraction of Chrysanthemum morifolium led to the isolation and purification of two compounds, namely morifonoside A (I) and chlorogenic acid (II), among which morifonoside A was one new flavonoid glycoside. The structure of morifonoside A was characterized as 4',5-dihydroxy-3',5'-dimethoxy flavone 7-O- $\beta$-D-glucoside on the spectral analysis of ${ }^{1} \mathrm{D}$ and ${ }^{2} \mathrm{D}$ NMR data.
\end{abstract}

Key Words: Chrysanthemum morifolium, Morifonoside A.

Chrysanthemum morifolium, one of the eight famous kinds of traditional Chinese medicine in Zhejiang Province, is a traditional agricultural medicinal plant widely used in China, Japan and Korea for pacifying living and improving eyesight. Modern pharmacological researches had also revealed that $C$. morifolium possessed the bioactivities of antianaphylaxis, antitumor, antimutagenesis and decompression, etc. ${ }^{1-4}$. A systematic chemical study of $C$. morifolium resulted in the isolation of two compounds, among, which one was a new flavonoid glycoside. Structural elucidation of the new compound was carried out through spectral methods and reported in this article.

Herbal material: Dried plant of $C$. morifolium was purchased in Tongxiang city, Zhejiang Prov., China. Voucher specimen had been identified by pharmacognosist Zengxi Guo and also been kept under certain conditions for future identification.

Melting point was carried out with the capillary tube method, without the calibration of temperature. NMR data were obtained by Bruker ARX-500. IR was carried out with Bruker IFS-55. High resolution MS was carried out with Bruker micrOTOFQ 125. All used reagents were of analytical grade and purchased from Hanbon Sci. and Tech Co. Ltd., (Huaian, Jiangshu Prov.).

Isolation procedure: The dried powder of $C$. morifolium $(500 \mathrm{~g})$ was refluxed with $70 \% \mathrm{EtOH}$ for $1 \mathrm{~h}$, then the extract was concentrated and suspended in $\mathrm{H}_{2} \mathrm{O}$. After partition with
$\mathrm{CHCl}_{3}$, EtOAc and $n$-BuOH. The $\mathrm{H}_{2} \mathrm{O}$ layer then underwent isolation by $\mathrm{D}-101$ macroporous resin, with gradient elution of $\mathrm{MeOH}-\mathrm{H}_{2} \mathrm{O}$ (1:10-10:1), giving 10 fractions. Fraction 3 was then isolated on $\mathrm{C}_{18}$ open column and further purified with Sephadex LH-20 and preparative RP HPLC, which finally yielded two compounds, morifonoside A (I, $3.2 \mathrm{mg}$ ) and chlorogenic acid (II, $15.4 \mathrm{mg}$ ).

Compound morifonoside A (I) (Fig. 1), yellow amorphous powder, had the melting point at $185-187^{\circ} \mathrm{C}$. The HR MS showed that I had the $\mathrm{m} / \mathrm{z} 493.1349\left([\mathrm{M}+\mathrm{H}]^{+}\right.$, calc. 493.1340$)$ and $515.1168\left([\mathrm{M}+\mathrm{Na}]^{+}\right.$, calc. 515.1160$)$, indicating the molecular formula to be $\mathrm{C}_{23} \mathrm{H}_{24} \mathrm{O}_{12}$. In the ${ }^{1} \mathrm{H}$ NMR spectrum (Table-1), the aromatic protons signals at $\delta 6.32(1 \mathrm{H}, \mathrm{d}, J=$ $2.1 \mathrm{~Hz}, \mathrm{H}-6)$ and $6.81(1 \mathrm{H}, \delta, J=2.1 \mathrm{~Hz}, \mathrm{H}-8)$ indicated the presence of a AB coupling system, while 7.21 (2H, s, H-2', 6') showed a typical $\mathrm{A}_{2}$ coupling system. Combining with the proton single at $\delta 6.97(1 \mathrm{H}, \mathrm{s}, \mathrm{H}-3)$, a flavone structure could be ensured. The proton signal at $\delta 3.66\left(6 \mathrm{H}, \mathrm{s},-\mathrm{OCH}_{3}\right)$ exhibited a single peak, revealing the existence of two methoxyls and these two methoxyl groups could only be assigned to the positions $3^{\prime}$ and 5' of $\mathrm{C}$ ring in flavone, instead of 3' and 4', otherwise the single peak of $\mathrm{H}-2^{\prime}, 6^{\prime}$ would be interfered to generate a split and form two doublet peaks. The signal at $\delta$ $5.01(1 \mathrm{H}, \mathrm{d}, J=6.9 \mathrm{~Hz}, \mathrm{H}-1 ")$ belonged to the anomeric proton of glycoside and the anomeric configuration was deduced to be $\beta$ according to the coupling constant $(6.9 \mathrm{~Hz})$. 


\begin{tabular}{|c|c|c|}
\hline \multicolumn{3}{|c|}{$\begin{array}{c}\text { TABLE-1 } \\
{ }^{1} \mathrm{H} \text { AND }{ }^{13} \mathrm{C}-\mathrm{NMR} \text { DATA FOR } \\
\text { MORIFONOSIDE A }\left(500 \mathrm{MHz}, \mathrm{ppm} \text { in } \mathrm{D}_{2} \mathrm{O}\right)\end{array}$} \\
\hline & ${ }^{1} \mathrm{H}-\mathrm{NMR}(\mathrm{J}$ in $\mathrm{Hz})$ & ${ }^{13} \mathrm{C}-\mathrm{NMR}$ \\
\hline \multicolumn{3}{|l|}{ Aglycone } \\
\hline 2 & & 162.3 \\
\hline 3 & $6.97(1 \mathrm{H}, \mathrm{s})$ & 101.6 \\
\hline 4 & & 179.3 \\
\hline 5 & & 156.4 \\
\hline 6 & $6.32(1 \mathrm{H}, \mathrm{d}, J=2.1)$ & 101.2 \\
\hline 7 & & 161.7 \\
\hline 8 & $6.81(1 \mathrm{H}, \mathrm{d}, J=2.1)$ & 100.5 \\
\hline 9 & & 155.4 \\
\hline 10 & & 105.9 \\
\hline $1^{\prime}$ & & 121.4 \\
\hline $2^{\prime}$ & $7.21(1 \mathrm{H}, \mathrm{s})$ & 103.9 \\
\hline 3 ' & & 146.5 \\
\hline 4 ' & & 141.6 \\
\hline 5 & & 146.5 \\
\hline 6 & $7.21(1 \mathrm{H}, \mathrm{s})$ & 103.9 \\
\hline $\mathrm{OCH}_{3}-3,5$, & $3.66(6 \mathrm{H}, \mathrm{s})$ & 56.1 \\
\hline \multicolumn{3}{|l|}{ Gly } \\
\hline 1, & $5.01(1 \mathrm{H}, \mathrm{d}, J=6.9)$ & 104.1 \\
\hline 2, & $3.13(1 \mathrm{H}, \mathrm{m})$ & 71.6 \\
\hline $3^{\prime \prime}$ & $3.34(1 \mathrm{H}, \mathrm{m})$ & 74.5 \\
\hline 4, & $3.18(1 \mathrm{H}, \mathrm{m})$ & 70.6 \\
\hline 5, & $3.45(1 \mathrm{H}, \mathrm{m})$ & 79.4 \\
\hline 6", & $3.37(2 \mathrm{H}, \mathrm{m})$ & 61.3 \\
\hline
\end{tabular}

The ${ }^{13} \mathrm{C}$ NMR signals (Table-1) also provided the evidence of flavone structure with methoxyl groups and glucosylation. HMQC spectrum helped to distinguish out the protons to each carbon, respectively. The HMBC correlation from $\mathrm{H}-1$ " to $\mathrm{C}-7$ revealed that the sugar moiety was linked to the aglycone position at $\mathrm{C}-7$ and correlations from $3^{\prime}-\mathrm{OCH}_{3}$ to $\mathrm{C}-\mathrm{2}^{\prime}, \mathrm{4}^{\prime}$ and 5'-OCH $\mathrm{OCH}_{3}$ to $\mathrm{C}-4$ ', 6' confirmed the conjunction positions of the two methoxyl groups. In the NOESY spectrum, the correlations of H-1" to H-6 and H-8, 3'-OCH ${ }_{3}$ to $\mathrm{H}-2$ ' and 5'-OCH 3 to H-6' further strengthened the confirmation about ascription results. Therefore, I was elucidated to be 4',5-dihydroxy-3',5'dimethoxy flavone 7-O- $\beta$-D-glucoside and named as morifonoside A. The key correlations of HMBC and NOESY were shown in Fig. 2.

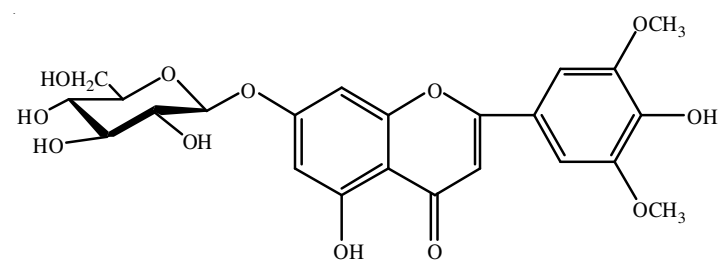

Fig. 1. Structure of morifonoside A

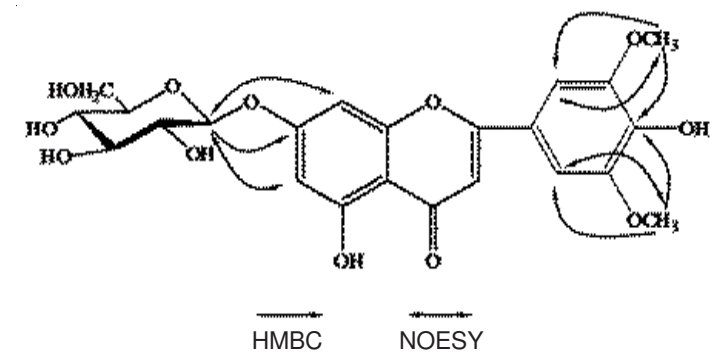

Fig. 2. Selected HMBC and NOESY correlations of morifonoside A

\section{REFERENCES}

1. J. Zhang, Y.B. Li, D.W. Qian, Y.X. Xie, Y.C. Wang and S.B. He, Lishizhen Medicined Materia Med. Res., 17, 1941 (2006).

2. X.Y. Zhang, L.H. Duan, D. Zhao and Y.F. Li, Lishizhen Medicined Materia Med. Res., 19, 1702 (2008).

3. X.Q. Wang, Y.B. Yue, Q.Y. Wang, L.Q. Song and Z.Q. Wang, Modern Food Sci. Tech., 27, 299 (2011).

4. X.Y. Sun, H.M. Shen, X.Q. Zhu, Z.Y. He and Y.Q. Hu, Chin. Trad. Herb. Drug, 39, 148 (2008). 